\title{
A case of radial keratoneuritis in non-Acanthamoeba keratitis
}

This article was published in the following Dove Press journal:

Clinical Ophthalmology

20 September 2012

Number of times this article has been viewed

\section{Tetsuya Mutoh \\ Yukihiro Matsumoto \\ Makoto Chikuda \\ Department of Ophthalmology, Dokkyo Medical University Koshigaya \\ Hospital, Saitama, Japan}

Correspondence: Tetsuya Mutoh Department of Ophthalmology, Dokkyo Medical University Koshigaya Hospital, 2-I-50 Minami-Koshigaya, Koshigaya, Saitama 343-8555, Japan

Tel +8| 48965 I I I I

Fax +8। 48965 । I 27

Email mtetsuya@dokkyomed.ac.jp
Abstract: A case of non-Acanthamoeba keratitis with radial keratoneuritis, which is thought to be pathognomonic for Acanthamoeba keratitis, is reported. A healthy 32-year-old woman with a history of frequent replacement of her contact lenses due to wear was examined at Dokkyo Medical University Koshigaya Hospital (Saitama, Japan) and found to have a slight corneal opacity that was accompanied by radial keratoneuritis. Based on both the patient's clinical findings and past history, the presence of Acanthamoeba keratitis was highly suspected. However, direct light microscopy of corneal scrapings stained by the Parker ink-potassium hydroxide method only found Acanthamoeba-type material in the specimen collected at her initial visit. In all other specimens collected from the patient, no Acanthamoeba was found either when using the same method or when performing cultures of the surgical debridement of the corneal lesion. In addition, topical antifungal eye drops, systemic antifungal drugs, and surgical debridement were also not effective in this case. Since a precise diagnosis could not be made, the patient was treated with topical $0.1 \%$ betamethasone sodium, which ultimately resulted in a dramatic improvement of her corneal inflammation. At 23 days after initiation of topical administration of $0.1 \%$ betamethasone sodium, visual acuity was $20 / 250$, with a slight corneal opacity noted at the original site of infection. The outcome of the current case suggests that radial keratoneuritis is not always pathognomonic for Acanthamoeba keratitis.

Keywords: radial keratoneuritis, non-Acanthamoeba keratitis, topical corticosteroid

\section{Introduction}

Acanthamoeba keratitis, which was first reported in $1974,{ }^{1}$ is often a severe, painful infection of the cornea that commonly causes corneal scaring and, in some cases, leads to blindness. ${ }^{2}$ Acanthamoeba keratitis is caused by the free-living amoeba Acanthamoeba, and is believed to be related to exposure to contaminated water. After a dramatic increase in the incidence of Acanthamoeba keratitis was found in contact lens wearers in the 1980s in the United States, it was subsequently determined that this change was associated with the use of contact lenses. ${ }^{3}$

Although radial keratoneuritis is a very important clinical sign, Acanthamoeba keratitis diagnosis is usually missed due to the fact the disease is so uncommon. ${ }^{4}$ Thus, if clinical progression of radial keratoneuritis is discovered in patients, the possibility of an Acanthamoeba keratitis diagnosis should also to be taken into consideration. ${ }^{5}$ Additionally, Moore et al have also reported that radial keratoneuritis is a characteristic sign of Acanthamoeba keratitis. ${ }^{6}$ However, there have also been reports of the occurrence of Pseudomonas keratitis with radial keratoneuritis and non-Acanthamoeba keratitis with radial keratoneuritis. ${ }^{7,8}$ 
Here, a case of radial keratoneuritis in infectious keratitis, which was suspected of being due to Acanthamoeba keratitis, is presented. The diagnosis was made based on clinical progress, inspection, and direct light microscopy of corneal specimens obtained during the patient's initial visit. Although initial findings suggested Acanthamoeba keratitis, direct light microscopy of the corneal specimens, culture isolation, and the effectiveness of the therapeutic drugs administered lead to the final diagnosis of non-Acanthamoeba keratitis with radial keratoneuritis.

\section{Case report}

A healthy 32-year-old woman presented at the Dokkyo Medical University Koshigaya Hospital (Saitama, Japan) on May 7, 2009 complaining of keratitis in her right eye. She frequently had to replace her contact lenses for correction of her myopia and she used a multipurpose solution for cleaning. She also washed her lens storage case using tap water. At the beginning of April 2009, she experienced hyperemia and sensation of a foreign body in her eye. These symptoms appeared to occur shortly after her frequent removal of the replacement contact lenses. Initially the patient was treated with topical antibiotics for a corneal abrasion at a contact lens clinic, but her symptoms did not resolve. In addition, another ophthalmologist treated her with a topical acyclovir eye ointment and $0.1 \%$ fluorometholone for presumed herpes simplex in the middle of April. However, her keratitis did not improve.

Upon initial examination, the patient's visual acuity was 20/40 (right eye). A slit-lamp examination of the right eye disclosed the presence of a mildly inflamed conjunctiva. The right cornea exhibited radial keratoneuritis at both the eleven and one o'clock positions, and there were several centrally located scattered subepithelial lesions that were approximately $3 \mathrm{~mm}$ in diameter (Figure 1). There was no reaction noted in the anterior chamber, ocular media, or ocular fundus. While Acanthamoeba keratitis was initially suspected, it was decided that a corneal biopsy specimen should be collected from the lesion for direct examination. Direct light microscopy of the specimen, which was stained by the Parker ink-potassium hydroxide method, revealed only the presence of Acanthamoeba-type material (Figure 2)., Also, bacterial culture was performed using a corneal biopsy specimen, but the result was negative and confocal microscopy was not performed.

The patient began a regimen of $200 \mathrm{mg}$ of oral itraconazole after breakfast once a day, in conjunction with an hourly administration of topical micafungin. She stopped

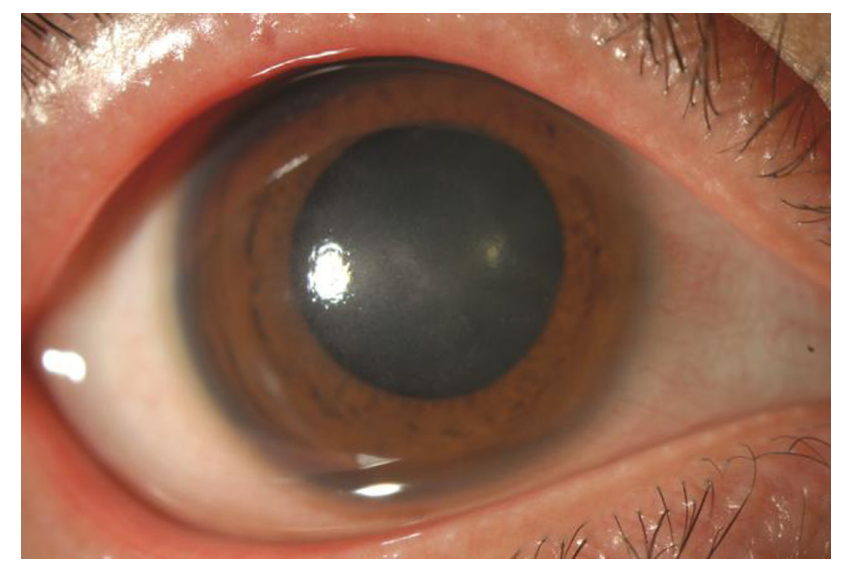

Figure I Photograph of a 32-year-old Japanese woman who exhibited radial keratoneuritis at the eleven and one o'clock positions in her right eye.

topical $0.1 \%$ fluorometholone from May 7 . She subsequently underwent surgical debridement of the lesion twice per week, for 3 weeks. However, neither the drug therapy nor the surgical intervention helped the patient, as both the conjunctival injection and pain increased, and there was an increase and deepening of the radial keratoneuritis (Figure 3). Direct examination of the specimens collected during the three surgical debridements on May 11, 14, and 18 revealed no other materials when using the Parker ink-potassium hydroxide method. On May 21, the corneal stroma was cultured using nonnutrient agar. No materials were found for these cultures. After visiting another ophthalmologist, topical acyclovir eye ointment five times per day was added to the therapeutic regimen. Even with the additional treatment, there was a further widening of the corneal lesion (Figure 4). Due to the lack of success with the previous treatments, topical $0.1 \%$ betamethasone sodium was subsequently added four times per day starting on July 1. Five days after initiation of

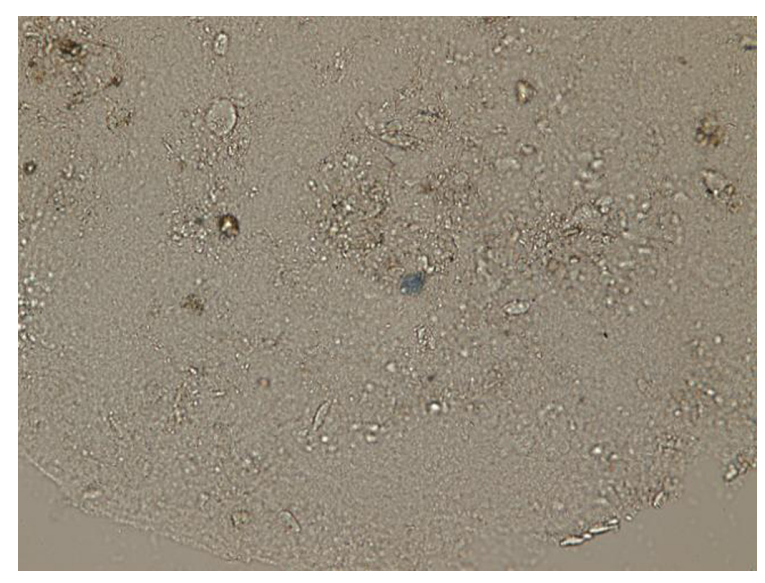

Figure 2 Direct examination of the specimens using the Parker ink-potassium hydroxide method revealed blue staining of Acanthamoeba material. 


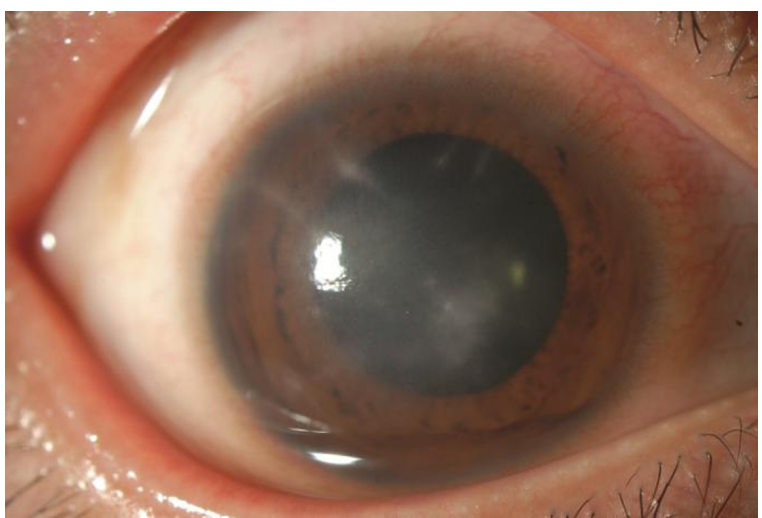

Figure 3 As the inflammation progressed, radial keratoneuritis was further observed at the eight, nine, ten, eleven, one, and three o'clock locations.

the topical $0.1 \%$ betamethasone sodium, the corneal lesion dramatically improved, with a total reepithelialization of the cornea noted. Because of the improvement, administration of the acyclovir eye ointment was stopped and the oral itraconazole was reduced to $100 \mathrm{mg}$ per day. Visual acuity at 23 days after initiation of the topical $0.1 \%$ betamethasone sodium was $20 / 250$ and, at that time, the cornea had no observable epithelial defect. Although a dramatic improvement was noted in the patient, a mild central corneal opacity still remained at the site of original infection at the end of the treatment period (Figure 5). However, since this time, she has not required any further treatments at the hospital.

\section{Discussion}

At the present time, there have only been two reported cases of non-Acanthamoeba keratitis with radial keratoneuritis. ${ }^{7,8}$ Feist et al reported being able to diagnose patients based on positive Pseudomonas aeruginosa corneal scraping

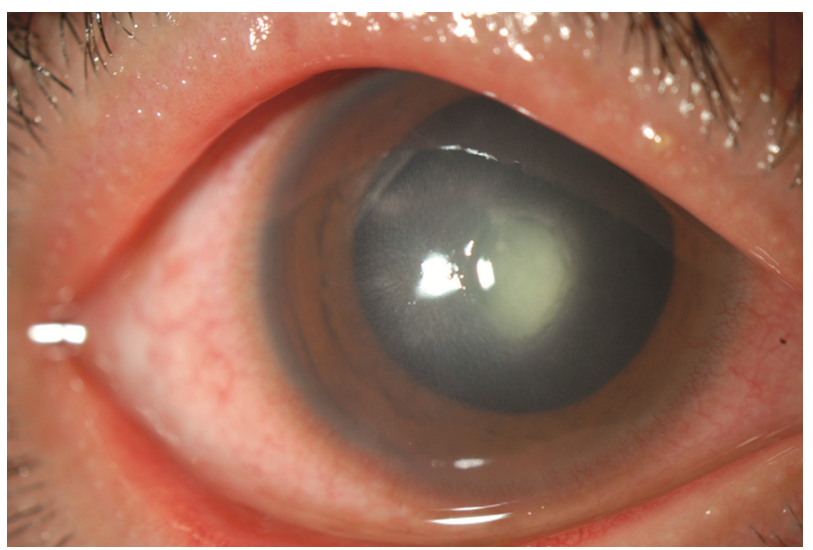

Figure 4 Ultimately, the continued progression of the inflammation led to ulceration in the center of the cornea.

Note: At this point, the radial keratoneuritis had almost completely disappeared.

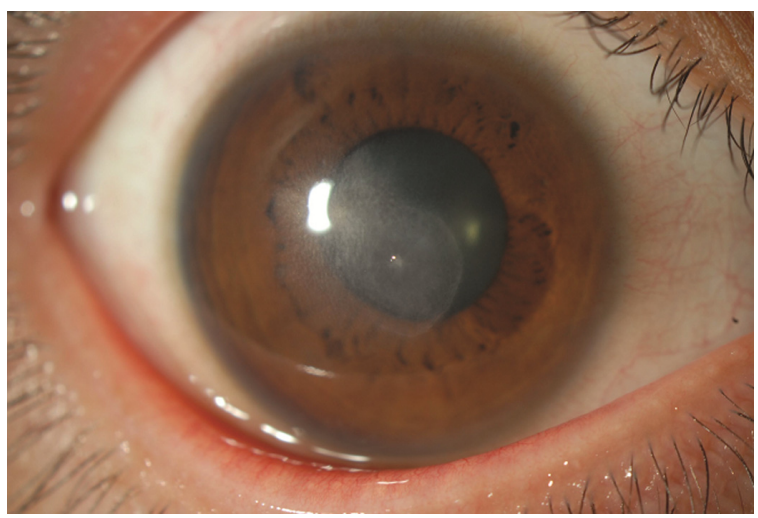

Figure 5 Even though the therapeutic intervention finally cured the inflammation, corneal opacity remained at the original site of the infection.

culture results. ${ }^{7}$ However, Shinoda et al have reported that many of these of examinations do not show the presence of any bacteria, fungi, or Acanthamoeba. Although antifungal drugs were only used for a short period of time, the keratitis was cured. ${ }^{8}$ Similar to past reports, the current results also indicate that radial keratoneuritis is not always pathognomonic for Acanthamoeba keratitis.

There have been many previous studies that have suggested that Acanthamoeba keratitis is involved in these types of cases, which include radial keratoneuritis, patients who require frequent replacement of their contact lenses, use of multipurpose solutions, and inadequate contact lenses care. Although there are no reports that have shown that topical antifungal eye drops, systemic antifungal drugs, or surgical debridement are not effective, it has only been definitively demonstrated that topical $0.1 \%$ betamethasone sodium is effective for Acanthamoeba keratitis. In the current case, the Acanthamoeba material was only found in the initial visit corneal biopsy specimen, but there have been many reports of Acanthamoeba being found in other cases. ${ }^{9-11}$ However, it should be noted that the Acanthamoeba material that was found in the initial visit specimen in the current case might actually not be Acanthamoeba, as further direct examinations of the specimen and culture were unable to find any conclusive evidence of Acanthamoeba. Regardless, if these symptoms are due to a bacterial infection, then the administration of topical levofloxacin eye drops should be effective in these types of cases. Since topical $0.1 \%$ betamethasone sodium proved to be dramatically effective in the current case, the stroma type of herpetic keratitis was suspected in this patient. ${ }^{12}$ Although corneal perception and viral cultures were not measured in the current case, this measurement should be performed as it has been shown to be useful when diagnosing the stroma type of herpetic keratitis. ${ }^{12}$ 
It has been demonstrated that Acanthamoeba grows best on horse blood agar and on nonnutrient agar seeded with an overlay of dead or living Gram-negative rods. ${ }^{4}$ It has also been found that Acanthamoeba organisms are less commonly isolated on other media, such as chocolate agar, Sabouraud agar, and Lowenstein-Jensen agar. Acanthamoeba are cultured at room temperature, with the trophozoites seen 5 days later, followed by the cysts 7 days later. ${ }^{13}$ When Acanthamoeba was cultured on nonnutrient agar that was seeded with Bacillus subtilis natto as the bait, the current results were negative. In addition, Acanthamoeba keratitis also appeared to be negative at this particular time point.

Topical polyhexamethylene biguanide and propamidine treatment are effective for this keratitis, ${ }^{4}$ but topical micafungin is also effective..$^{10,11,14,15}$ Because it is difficult to obtain polyhexamethylene biguanide and propamidine in Japan, micafungin was used. Topical corticosteroids might mask characteristic findings of Acanthamoeba keratitis, ${ }^{14}$ and were avoided as much as possible.

\section{Conclusion}

It has been demonstrated that radial keratoneuritis occurs during the early stage of Acanthamoeba keratitis, a characteristic that is useful for diagnosis. ${ }^{14,15}$ Although these types of cases are very rare, one must be careful when interpreting clinical results as cases of non-Acanthamoeba keratitis with radial keratoneuritis do exist.

\section{Disclosure}

The authors report no conflicts of interest in this work.

\section{References}

1. Nagington J, Watson PG, Playfair TJ, McGill J, Jones BR, Steele AD. Amoebic infection of the eye. Lancet. 1974;2(7896):1537-1540.

2. Joslin CE, Tu EY, McMahon TT, Passaro DJ, Stayner LT, Sugar J. Epidemiological characteristics of a Chicago-area Acanthamoeba keratitis outbreak. Am J Ophthalmol. 2006;142(2):212-217.

3. Moore MB, McCulley JP, Luckenbach M, et al. Acanthamoeba keratitis associated with soft contact lenses. Am J Ophthalmol. 1985;100(3): 396-403.

4. IshibashiY.Acanthamoeba keratitis. Ophthalmologica. 1997;211(Suppl 1): 39-44.

5. IshibashiY, Kamei Y, Nyunt AK, et al. Radial keratoneuritis as the initial clinical manifestation in two cases of Acanthamoeba keratitis. Rinsho Ganka. 1996;50:1881-1885. Japanese.

6. Moore MB, McCulley JP, Kaufman HE, Robin JB. Radial keratoneuritis as a presenting sign in Acanthamoeba keratitis. Ophthalmology. 1986;93(10):1310-1315.

7. Feist RM, Sugar J, Tessler H. Radial keratoneuritis in Pseudomonas keratitis. Arch Ophthalmol. 1991;109(6):774-775.

8. Shinoda K, Kobayashi A, Miwa S, et al. A case of non-Acanthamoeba keratitis with radial keratoneuritis. Nihon Ganka Kiyo. 2002;53: 894-897. Japanese.

9. Mutoh T, Segawa A, Itoh T, et al. Two cases of severe corneal damage in frequent replacement contact lenses wearers. Ganka. 2009;51: 191-197. Japanese.

10. Ishikawa I, Mutoh T, Matsumoto Y, et al. Three cases of Acanthamoeba keratitis treated by topical administration of micafungin and azole type antifungal drugs. Ganka. 2010;52:1087-1092. Japanese.

11. Mutoh T, Ishikawa I, Matsumoto Y, Chikuda M. A retrospective study of nine cases of Acanthamoeba keratitis. Clin Ophthalmol. 2010;4:1189-1192.

12. Inoue Y. Diagnosis and treatment of herpetic keratitis. Nihon Ganka Gakkai Zasshi. 2008;112:83-93. Japanese.

13. Ishibashi Y. Contact lenses and Acanthamoeba. J Jpn Contact Lens Soc. 1996;38:237-241. Japanese.

14. Sasaki M, Sotozono C, Chihara H, et al. Characteristic appearance of early-stage Acanthamoeba keratitis. Nihon Ganka Gakkai Zasshi. 2010;114(12):1030-1035. Japanese.

15. Matsumoto Y, Dogru M, Sato EA, et al. The application of in vivo confocal scanning laser microscopy in the management of Acanthamoeba keratitis. Mol Vis. 2007;13:1319-1326.
Clinical Ophthalmology

\section{Publish your work in this journal}

Clinical Ophthalmology is an international, peer-reviewed journal covering all subspecialties within ophthalmology. Key topics include: Optometry; Visual science; Pharmacology and drug therapy in eye diseases; Basic Sciences; Primary and Secondary eye care; Patient Safety and Quality of Care Improvements. This journal is indexed on Submit your manuscript here: http://www.dovepress.com/clinical-ophthalmology-journal

\section{Dovepress}

PubMed Central and CAS, and is the official journal of The Society of Clinical Ophthalmology (SCO). The manuscript management system is completely online and includes a very quick and fair peer-review system, which is all easy to use. Visit http://www.dovepress.com/ testimonials.php to read real quotes from published authors. 